\title{
SYNTHESIS AND THE ANTIMICROBIAL ACTIVITY OF ETHYL 5-METHYL-2-(ALKYLTHIO)-4-OXO-3,4-DIHYDROTHIENO [2,3-d]PYRIMIDINE-6-CARBOXYLATES
}

\author{
O.V.Tkachenko, S.V.Vlasov, S.M.Kovalenko, I.O.Zhuravel', V.P.Chernykh \\ National University of Pharmacy \\ 53, Pushkinska str., Kharkiv, 61002, Ukraine. E-mail: kosn@ukrfa.kharkov.ua
}

Key words: thiophene; pyrimidine; thiourea

\begin{abstract}
By alkylation of the products of diethyl 3-methyl-5-\{[(methylthio)carbonothioyl]amino\}-2,4-thiophenedicarboxylate interaction with benzylamines the novel derivatives of ethyl 5-methyl-2-(alkylthio)-4-oxo-3,4-dihydrothieno[2,3-d]pyrimidine-6-carboxylates have been obtained. It has been found that the signal of the $\mathrm{CH}_{2}$-group adjacent to the nitrogen atom in position 3 of thieno[2,3-d]pyrimidine system is always observed in the range of 5.35-5.40 ppm, while the position of the signal of methythylene-group connected with the sulfur atom much depends upon the structure of the radical attached to this group. IR-spectra of all the compounds contain the intensive $C=O$ stretching band at $1721-1678 \mathrm{~cm}^{-1}$; the spectra of the compounds with amide function contain bands of stretching $\mathrm{N}-\mathrm{H}$ of $3280-3263 \mathrm{~cm}^{-1}$, while nitriles have the band of stretching $C \equiv N$ vibrations near $2250 \mathrm{~cm}^{-1}$. It has been determined that all of the compounds are mostly active against the strain of Candida aibicans fungi. The most resistant microorganism was found to be the strains of Staphylococcus aureus. The only exception is the derivative modified with the thioacetic acid residue in position 2 and unsubstituted benzyl in position 3, which appeared to be highly active against Staphylococcus aureus strain. Amides of thioactetic acid modified in position 3 with 3,4-dichlorobenzyl substituent and thioacetamide substituents in position 2 are active against Pseudomonas aeruginosa, as well as the compound, which contains 3-chlorobenzyl substituent in position 3 and p-chlorobenzotiol substituents in position 2 of thieno[2,3-d]pyrimidine.
\end{abstract}

СИНТЕЗ ТА АНТИМІКРОБНА АКТИВНІСТЬ ПОХІДНИХ ЕТИЛ 5-МЕТИЛ-2-(АЛКІЛТІО)-4-ОКСО-3,4-ДИГІДРОТІЄНО[2,3-d]ПІРИМІДИН-6-КАРБОКСИЛАТІВ

О.В.Ткаченко, С.В.Власов, С.М.Коваленко, І.О.Журавель, В.П.Черних

Ключові слова: тіофен; піримідин; тіосечовина

Шляхом алкілування продуктів взаємодії діетил 3-метил-5-\{[(метилтіо)карбонотіоїл]аміно\}-2,4-тіофрендикарбоксилату з бензиламінами отримані нові похідні етил 5-метил-2-(алкілтіо)-4-оксо-3,4-дигідротієно[2,3-d] піримідин-6-карбоксилатів. Встановлено, що в спектрах ${ }^{1} \mathrm{H}$ ЯМР сигнал групи $\mathrm{CH}_{2}$, з'єднаної з атомом Нітрогену у положенні 3 тієно[2,3-d]піримідинової системи, для усіх кінцевих сполук знаходиться в діапазоні 5,35-5,4 м.ч., в той же час положення сигналу метиленової групи біля атома Сульфуру значно залежить від будови радикалу, безпосередньо зв'язаного з нею. В ІЧ-спектрах для усіх сполук характерними $є$ інтенсивні смуги валентних коливань C=O 1721-1678 см-1. для сполук з амідним фррагментом наявні смуги валентних коливань $\mathrm{N}-\mathrm{H}$ 3280-3263 cм-1, для нітрилів спостерігається смуга валентних коливань CइN при $2250 \mathrm{~cm}^{-1}$. Встановлено, що всі сполуки найбільше пригнічують ріст грибів Candida aibicans. Найменш активною більшість сполук виявилась до штамів Staphylococcus aureus. Виключенням є лише сполука із залишком тіооцтової кислоти у положенні 2 та незаміщеним бензильним замісником у положенні 3, яка проявила значну активність по відношенню до штаму Staphylococcus aureus. Aміди miооитової кислоти з 3,4-дихлоробензильним замісником у положенні 3 та тіоацетамідними замісниками у положенні $2 \epsilon$ активними по відношенню до Pseudomonas aeruginosa, так як і сполука, що містить 3-хлоробензильний замісник у положенні 3 тієно[2,3-d]піримідину та п-хлоробензотіольний замісник у положенні 2.

СИНТЕЗ И ПРОТИВОМИКРОБНАЯ АКТИВНОСТЬ ПРОИЗВОДНЫХ ЭТИЛ 5-МЕТИЛ-2-(АЛКИЛТИО)-4ОКСО-3,4-ДИГИДРОТИЕНО[2,3-d]ПИРИМИДИН-6-КАРБОКСИЛАТОВ

Е.В.Ткаченко, С.В.Власов, С.Н.Коваленко, И.А.Журавель, В.П.Черных

Ключевые слова: тиофен; пиримидин; тиомочевина

Путем алкилирования продуктов взаимодействия диэтил 3-метил-5-\{[(метилтио)карбонотиоил]амино\}2,4-тиофендикарбоксилата с бензиламинами получены новые производные этил 5-метил-2-(алкилтио)4-оксо-3,4-дигидротиено[2,3-d]пиримидин-6-карбоксилатов. Установлено, что в спектрах ${ }^{1}$ Н ЯМР сиटнал группы $\mathrm{CH}_{2}$, связанной с атомом азота в положении 3 тиено[2,3-d]пиримидиновой системы, для всех конечных соединений находится в диапазоне 5,35-5,4 м.д., в то же время положение сигнала метиленовой группы возле атома серы значительно зависит от строения радикала, непосредственно связанного с ней. В ИК-спектрах для всех соединений характерными являются интенсивные полосы валентных колебаний C=O 1721-1678 см-1, для соединений с амидным фрагментом присутствуют полосы валентных колебаний $\mathrm{N}-\mathrm{H}$ 3280-3263 см-1, для нитрилов наблюдается полоса валентных колебаний CइN при $2250 \mathrm{~cm}^{-1}$. Установлено, что все соединения наиболее активно подавляют рост грибов Candida aibicans. Большинство соединений оказалось наименее активным по отношению к штаммам Staphylococcus aureus. Исключением из этого является соединение с остатком тиоуксусной кислоты в положении 2 и незамещенным бензильным заместителем в положении 3, которое проявило значительную активность по отношению к штамму Staphylococcus aureus. Амиды тиоуксусной кислоты с 3,4-дихлорбензильным заместителем в положении 3 и тиоацетамидными заместителями в положении 2 являются активными по отношению к Pseudomonas aeruginosa так же как и соединение, содержащее 3-хлорбензильный заместитель в положении 3 тиено[2,3-d]пиримидина и п-хлорбензотиольный заместитель в положении 2. 
It has been recently published that esters of 5methyl-2-thioxothieno[2,3-d]pyrimidine-6-carboxylic acid display affinity to histamine receptors of $5-\mathrm{HT}_{3}$ subtype and could be used as medicines for treatment of Bowel disease. They may also bind 5- $\mathrm{HT}_{1 \mathrm{~A}}$ subtype receptors, which make them useful for treatment of stress induced ulcer [1]. As the result of in vivo investigation of S-glycosides of ethyl ester of 3amino-5-methyl-2-thioxothieno[2,3-d]pyrimidine-6carboxylic acid some compounds with a high range of anti-inflammatory activity have been found [2]. Esters of 2-S-alkylthiothieno[2,3-d]pyrimidine-6-carboxylic acid may also be applied as the intermediates for preparation of fused systems of heterocycles with the antimicrobial activity [3].

Since the number of works dedicated to investigations of the biological activity of 5-methyl-2-thioxothieno[2,3-d]pyrimidine-6-carboxylic acid is not enough, we devoted our work to the synthesis and research of the antimicrobial activity of some derivatives of ethyl 5-methyl-2-(alkylthio)-4-oxo-3,4-dihydrothieno [2,3-d]pyrimidine-6-carboxylate.

Synthesis of the target compounds has been carried out by cyclization of the starting dithiocarbamate $\mathbf{1}[4,5]$, with primary, amines [4]. Mostly amines of benzyl type were used. The above mentioned procedure resulted in the corresponding 3-R-5-methyl4-oxo-2-thioxo-1,2,3,4-tertrahydrothieno[2,3-d]pyrimidine-6-carboxylates 2 as the crude products, which were suitable for further use without additional purification.

Target molecules $\mathbf{3}$ were prepared by alkylation of intermediates $\mathbf{2}$ with different alkyl halides (like benzyl chlorides and derivatives of chloroacetic acid). The radicals $\mathrm{R}$ and $\mathrm{R}^{1}$ and physicochemical properties of compounds $\mathbf{3}$ are listed in Table 1.

The structures of all compounds $\mathbf{3}$ were determined by ${ }^{1} \mathrm{H}$ NMR and IR-spectral data (Tables 2 and 3 ). In all ${ }^{1} \mathrm{H}$ NMR-spectra of compounds 3 the signal of the methyl group is observed in the range of 2.65$2.85 \mathrm{ppm}$ together with the signals of the carbethoxygroup protons as a triplet of $\mathrm{CH}_{3}$ at $1.15-1.38 \mathrm{ppm}$ and a quartet of $\mathrm{CH}_{2}$-group at 4.2- $4.6 \mathrm{ppm}$. The signal of the $\mathrm{CH}_{2}$-group adjacent to the nitrogen-atom in position 3 of thieno[2,3-d]pyrimidine system is always observed in the range of 5.35-5.40 ppm. The position of the methythylene-group attached to the atom of sulfur much depends upon the structure of $\mathrm{R}^{1}$; for example, for benzylated derivatives its position is located in the range of 4.43-4.47 ppm, for compounds with the benzoyl-group attached to $\mathrm{CH}_{2}$ it is shifted downfield to $4.78-4.85 \mathrm{ppm}$. For compounds $\mathbf{3 h}, \mathbf{3 k}$, $\mathbf{3 p}$ and $\mathbf{3 q}$ with the carbethoxy-group this signal is found at 3.8-4.05 ppm, nitriles $3 \mathrm{~g}$ and $3 \mathbf{m}-4.20$ 4.35 ppm, carboxamides 3d, 31, 3r-3u - 3.85-4.1 ppm; for acid 3e the signal $\mathrm{CH}_{2} \mathrm{COOH}$ is located at $4.03 \mathrm{ppm}$. For compounds $\mathbf{3 t}$ and $\mathbf{3} \mathbf{u}$ with the phenylethyl fragment two signals of methylene-groups are present $4.2\left(2 \mathrm{H}, \mathrm{t}\right.$., $\left.\mathrm{NC}_{2} \mathrm{CH}_{2} \mathrm{Ph}\right)$ and $3.03\left(2 \mathrm{H} \mathrm{t}\right.$., $\left.\mathrm{NCH}_{2} \mathrm{CH}_{2} \mathrm{Ph}\right)$. The integral intensity of all the signals present in the region the aromatic protons resonance well correlates with the number of protons in the structure of compounds 3 . For compound $3 e$ the signal of the carboxyl-group OH-proton is observed at $12.8 \mathrm{ppm}$; amides $\mathbf{3 d}, \mathbf{3 l}, \mathbf{3 r} \mathbf{r} \mathbf{3} \mathbf{u}$ show the signals of $\mathrm{NH}$-proton in the range of 7.9-8.7 ppm.

IR-spectra of all compound 3 show the stretching bands of $\mathrm{C}-\mathrm{H}$ in the region of $3091-2853 \mathrm{~cm}^{-1}$; for all of these compounds the intensive $\mathrm{C}=0$ stretching band is present at $1721-1678 \mathrm{~cm}^{-1}$. The spectra of compounds 3 with the amide function contain bands of stretching $\mathrm{N}-\mathrm{H} 3280-3263 \mathrm{~cm}^{-1}$, while nitriles have the band of stretching $\mathrm{C} \equiv \mathrm{N}$ vibrations near $2250 \mathrm{~cm}^{-1}$.

The antimicrobial activity of compound 3 were investigated by the agar well diffusion method $[6,7]$. The antimicrobial effect was measured by the diameter of the growth inhibition zone based on the known data about active antibiotics applied for the well diffusion method against susceptible microorganism strains $[7,8]$. To evaluate the antimicrobial activity the following criteria were used: in the case of the inhibition zone absence or its diameter was less than $10 \mathrm{~mm}$ either the bacteria strains were considered to be resistant or the concentration of the compound tested was rather low for the inhibition effect; the diameter of the inhibition zone was $10-15 \mathrm{~mm}$ - the low sensitivity of the bacteria strain to the compound in the given concentration; the diameter of the inhibition zone of 15-25 mm was considered as the sign of the substance activity against the microorganism strain; the diameter of the inhibition zone of $25 \mathrm{~mm}$ or more was considered as the evidence of the high antimicrobial activity of the compound tested. The results of the antimicrobial activity assay are presented in Table 4.

All of compounds $\mathbf{3}$ possessed the antimicrobial activity, but they were mostly active against the strain of Candida albicans fungi. The most antifungal activity was displayed by compounds 3e, 3o and 3s, which<smiles>[R]Sc1nc2sc(C(=O)OCC)c(C)c2c(=O)n1[R]</smiles> 
Table 1

Physicochemical properties of ethyl

5-methyl-2-(alkylthio)-4-oxo-3,4-dihydrothieno[2,3-d]pyrimidine-6-carboxylate derivatives 3

\begin{tabular}{|c|c|c|c|c|c|c|}
\hline \multirow[b]{2}{*}{ Compound } & \multirow[b]{2}{*}{$\mathrm{R}$} & \multirow[b]{2}{*}{$\mathrm{R}^{1}$} & \multirow{2}{*}{$\begin{array}{l}\text { Mol. formula } \\
\text { M.w. }\end{array}$} & \multirow{2}{*}{$\begin{array}{c}\text { Yield \%, } \\
\text { in the alkylation } \\
\text { step }\end{array}$} & \multirow[b]{2}{*}{ M.p., ${ }^{\circ} \mathrm{C}$} & $\mathrm{N} \%$ \\
\hline & & & & & & calc. \\
\hline $3 a$ & $\mathrm{Bn}$ & $\mathrm{CH}_{3}$ & $\begin{array}{c}\mathrm{C}_{18} \mathrm{H}_{18} \mathrm{~N}_{2} \mathrm{O}_{3} \mathrm{~S}_{2} \\
374.48\end{array}$ & 76 & 180 & $\begin{array}{l}7.48 \\
7.57\end{array}$ \\
\hline $3 b$ & $\mathrm{Bn}$ & $\mathrm{CH}_{2} \mathrm{COPh}$ & $\begin{array}{c}\mathrm{C}_{25} \mathrm{H}_{22} \mathrm{~N}_{2} \mathrm{O}_{4} \mathrm{~S}_{2} \\
478.59\end{array}$ & 87 & 159-161 & $\frac{5.85}{5.99}$ \\
\hline $3 c$ & $\mathrm{Bn}$ & $\mathrm{p}-\mathrm{ClBn}$ & $\begin{array}{c}\mathrm{C}_{24} \mathrm{H}_{21} \mathrm{CIN}_{2} \mathrm{O}_{3} \mathrm{~S}_{2} \\
485.03\end{array}$ & 85 & 137-139 & $\frac{5.78}{5.92}$ \\
\hline $3 d$ & $\mathrm{Bn}$ & $\mathrm{CH}_{2} \mathrm{CONH}$ (Cyclohexyl) & $\begin{array}{c}\mathrm{C}_{25} \mathrm{H}_{29} \mathrm{~N}_{3} \mathrm{O}_{4} \mathrm{~S}_{2} \\
499.66\end{array}$ & 94 & $261-262$ & 8.41 \\
\hline $3 e$ & $\mathrm{Bn}$ & $\mathrm{CH}_{2} \mathrm{COOH}$ & $\begin{array}{c}\mathrm{C}_{19} \mathrm{H}_{18} \mathrm{~N}_{2} \mathrm{O}_{5} \mathrm{~S}_{2} \\
418.49\end{array}$ & 61 & 192-193 & $\frac{6.69}{6.85}$ \\
\hline $3 f$ & $4-F B n$ & $\mathrm{CH}_{2} \mathrm{COPh}$ & $\begin{array}{c}\mathrm{C}_{25} \mathrm{H}_{21} \mathrm{FN}_{2} \mathrm{O}_{4} \mathrm{~S}_{2} \\
496.58\end{array}$ & 82 & $176-178$ & 5.64 \\
\hline $3 g$ & $4-F B n$ & $\mathrm{p}-\mathrm{ClBn}$ & $\begin{array}{c}\mathrm{C}_{24} \mathrm{H}_{20} \mathrm{ClFN}_{2} \mathrm{O}_{3} \mathrm{~S}_{2} \\
503.02\end{array}$ & 89 & $162-164$ & $\frac{5.57}{5.87}$ \\
\hline $3 \mathrm{~h}$ & $4-F B n$ & $\mathrm{CH}_{2} \mathrm{COOEt}$ & $\begin{array}{c}\mathrm{C}_{21} \mathrm{H}_{21} \mathrm{FN}_{2} \mathrm{O}_{5} \mathrm{~S}_{2} \\
464.54\end{array}$ & 70 & $177-179$ & $\frac{6.03}{6.17}$ \\
\hline $3 i$ & $2-\mathrm{ClBn}$ & $\mathrm{CH}_{2} \mathrm{COPh}$ & $\begin{array}{c}\mathrm{C}_{25} \mathrm{H}_{21} \mathrm{ClN}_{2} \mathrm{O}_{4} \mathrm{~S}_{2} \\
513.04\end{array}$ & 85 & $163-165$ & $\frac{5.46}{5.67}$ \\
\hline $3 g$ & $2-\mathrm{ClBn}$ & $\mathrm{CH}_{2} \mathrm{CN}$ & $\begin{array}{c}\mathrm{C}_{19} \mathrm{H}_{16} \mathrm{ClN}_{3} \mathrm{O}_{3} \mathrm{~S}_{2} \\
433.94\end{array}$ & 68 & $166-168$ & $\frac{9.68}{9.78}$ \\
\hline $3 \mathrm{k}$ & $2-\mathrm{ClBn}$ & $\mathrm{CH}_{2} \mathrm{COOEt}$ & $\begin{array}{c}\mathrm{C}_{21} \mathrm{H}_{21} \mathrm{ClN}_{2} \mathrm{O}_{5} \mathrm{~S}_{2} \\
480.99\end{array}$ & 73 & $128-130$ & $\frac{5.82}{5.95}$ \\
\hline 31 & $2-\mathrm{ClBn}$ & $\mathrm{CH}_{2} \mathrm{CONHBn}$ & $\begin{array}{c}\mathrm{C}_{26} \mathrm{H}_{24} \mathrm{CIN}_{3} \mathrm{O}_{4} \mathrm{~S}_{2} \\
542.08\end{array}$ & 93 & $175-176$ & $\frac{7.75}{7.92}$ \\
\hline $3 m$ & $3-\mathrm{ClBn}$ & $\mathrm{CH}_{2} \mathrm{CN}$ & $\begin{array}{c}\mathrm{C}_{19} \mathrm{H}_{16} \mathrm{ClN}_{3} \mathrm{O}_{3} \mathrm{~S}_{2} \\
433.94\end{array}$ & 62 & $183-185$ & $\frac{9.68}{9.98}$ \\
\hline $3 n$ & $3-\mathrm{ClBn}$ & $\mathrm{CH}_{2} \mathrm{COPh}$ & $\begin{array}{c}\mathrm{C}_{25} \mathrm{H}_{21} \mathrm{CIN}_{2} \mathrm{O}_{4} \mathrm{~S}_{2} \\
513.04\end{array}$ & 85 & 164-165 & $\frac{5.46}{5.55}$ \\
\hline 30 & $3-\mathrm{ClBn}$ & $\mathrm{p}-\mathrm{ClBn}$ & $\begin{array}{c}\mathrm{C}_{24} \mathrm{H}_{20} \mathrm{Cl}_{2} \mathrm{~N}_{2} \mathrm{O}_{3} \mathrm{~S}_{2} \\
519.47\end{array}$ & 78 & $140-145$ & $\frac{5.39}{5.67}$ \\
\hline $3 p$ & $3-\mathrm{ClBn}$ & $\mathrm{CH}_{2} \mathrm{COOEt}$ & $\begin{array}{c}\mathrm{C}_{21} \mathrm{H}_{21} \mathrm{ClN}_{2} \mathrm{O}_{5} \mathrm{~S}_{2} \\
480.99\end{array}$ & 82 & $152-155$ & $\frac{5.82}{5.93}$ \\
\hline $3 q$ & 3,4-diClBn & $\mathrm{CH}_{2} \mathrm{COOEt}$ & $\begin{array}{c}\mathrm{C}_{21} \mathrm{H}_{20} \mathrm{Cl}_{2} \mathrm{~N}_{2} \mathrm{O}_{5} \mathrm{~S}_{2} \\
515.44\end{array}$ & 88 & 191-193 & $\frac{5.43}{5.49}$ \\
\hline $3 r$ & 3,4-diClBn & $\mathrm{CH}_{2} \mathrm{CONHBn}$ & $\begin{array}{c}\mathrm{C}_{26} \mathrm{H}_{23} \mathrm{Cl}_{2} \mathrm{~N}_{3} \mathrm{O}_{4} \mathrm{~S}_{2} \\
576.52\end{array}$ & 93 & $215-217$ & $\frac{7.29}{7.44}$ \\
\hline $3 s$ & 3,4-diClBn & $\mathrm{CH}_{2} \mathrm{CONH}$ (Cyclohexyl) & $\begin{array}{c}\mathrm{C}_{25} \mathrm{H}_{27} \mathrm{Cl}_{2} \mathrm{~N}_{3} \mathrm{O}_{4} \mathrm{~S}_{2} \\
568.55\end{array}$ & 90 & $218-220$ & $\begin{array}{l}7.39 \\
7.49\end{array}$ \\
\hline $3 t$ & $\mathrm{CH}_{2} \mathrm{CH}_{2} \mathrm{Ph}$ & $\mathrm{CH}_{2} \mathrm{CONHBn}$ & $\begin{array}{c}\mathrm{C}_{27} \mathrm{H}_{27} \mathrm{~N}_{3} \mathrm{O}_{4} \mathrm{~S}_{2} \\
521.66\end{array}$ & 78 & $212-213$ & $\frac{8.06}{8.22}$ \\
\hline $3 u$ & $\mathrm{CH}_{2} \mathrm{CH}_{2} \mathrm{Ph}$ & $\mathrm{CH}_{2} \mathrm{CONH}($ Cyclohexyl) & $\begin{array}{c}\mathrm{C}_{26} \mathrm{H}_{31} \mathrm{~N}_{3} \mathrm{O}_{4} \mathrm{~S}_{2} \\
513.68\end{array}$ & 83 & 197-198 & $\frac{8.18}{8.35}$ \\
\hline
\end{tabular}

showed the most diameters of the growth inhibition zones. The most resistant microorganisms were determined to be the strains of Staphylococcus aureus and Proteus vulgaris. The only exception was the derivative $3 \mathbf{e}$ modified with the thioacetic acid residue in position 2 , which appeared to be highly active against
Staphylococcus aureus strain. Amides of thioactetic acid modified in position 3 with 3,4-dichlorobenzyl substituent $3 \mathbf{r}$ and $3 \mathbf{s}$ were active against Pseudomonas aeruginosa, as well as compound 30 with 3-chlorobenzyl substituents in position 3 and $p$-chlorobenzotiol substituents in position 2 . 
Data of ${ }^{1} \mathrm{H}$ NMR-spectra of ethyl

5-methyl-2-(alkylthio)-4-oxo-3,4-dihydrothieno[2,3-d]pyrimidine-6-carboxylate derivatives 3

\begin{tabular}{|c|c|c|c|c|c|}
\hline \multirow[b]{2}{*}{ Compound } & \multicolumn{5}{|c|}{ Chemical shift, $\delta$, ppm. } \\
\hline & $\begin{array}{l}\mathrm{NH} \\
\mathrm{OH}\end{array}$ & $\begin{array}{c}\mathrm{CH}_{3} \\
(3 \mathrm{H}, \mathrm{s} .)\end{array}$ & $\begin{array}{c}\mathrm{OCH}_{2} \mathrm{CH}_{3} \\
(3 \mathrm{H}, \mathrm{t} .+2 \mathrm{H} \text {, q. })\end{array}$ & aliphatic protons & aromatic protons \\
\hline 1 & 2 & 3 & 4 & 5 & 6 \\
\hline $3 a$ & - & 2.3 & $1.37+4.33$ & $\begin{array}{l}2.58\left(3 \mathrm{H}, \mathrm{t} ., \mathrm{SC}_{\mathrm{H}}\right) \\
5.31\left(2 \mathrm{H}, \text { s., } \mathrm{C}_{2} \mathrm{Ph}\right)\end{array}$ & $7.30(5 \mathrm{H}, \mathrm{m} ., \mathrm{Ar}-\mathrm{H})$ \\
\hline $3 b$ & - & 2.78 & $1.35+4.3$ & $\begin{array}{l}5.3\left(2 \mathrm{H}, \mathrm{s} ., \mathrm{NCH}_{2} \mathrm{Ph}\right) \\
4.85\left(2 \mathrm{H}, \mathrm{S}, \mathrm{SC}_{2} \mathrm{COPh}\right)\end{array}$ & $\begin{array}{l}7.30(5 \mathrm{H}, \mathrm{m} ., \mathrm{Ar}-\mathrm{H}) \\
7.55\left(2 \mathrm{H}, \mathrm{t.}, \mathrm{H}-5^{\prime}+\mathrm{H}-3^{\prime}\right) \\
7.68\left(1 \mathrm{H}, \mathrm{t.}, \mathrm{H}-4^{\prime}\right) \\
8.02\left(2 \mathrm{H}, \mathrm{d} ., \mathrm{H}-2^{\prime}+\mathrm{H}-6^{\prime}\right)\end{array}$ \\
\hline $3 c$ & - & 2.83 & $1.37+4.3$ & $\begin{array}{l}5.25\left(2 \mathrm{H}, \mathrm{s} ., \mathrm{CH}_{2} \mathrm{Ph}\right) \\
4.47\left(2 \mathrm{H}, \mathrm{s} ., \mathrm{SC}_{2}(4-\mathrm{ClPh})\right)\end{array}$ & $7.30(9 \mathrm{H}, \mathrm{m} ., \mathrm{Ar}-\mathrm{H})$ \\
\hline $3 d$ & $7.97(\mathrm{NH})$ & 2.83 & $1.3+4.3$ & $\begin{array}{l}\text { 1.1-1.8 (10 H, m., cyclohexyl) } \\
\left.\text { 3.89 (2H, s., } \mathrm{SC}_{2} \mathrm{CO}\right) \\
5.3\left(2 \mathrm{H}, \mathrm{s} ., \mathrm{NC}_{\mathrm{H}} \mathrm{Ph}\right) \\
3.52(1 \mathrm{H}, \mathrm{m} ., \mathrm{NH} \text { CHcyclohexyl) }\end{array}$ & $7.30(5 \mathrm{H}, \mathrm{m} ., \mathrm{Ar}-\mathrm{H})$ \\
\hline $3 e$ & $12.8(\mathrm{OH})$ & 2.85 & $1.22+4.32$ & $\begin{array}{l}4.03\left(2 \mathrm{H}, \mathrm{s} ., \mathrm{SCH}_{2} \mathrm{COOH}\right) \\
5.25\left(2 \mathrm{H}, \text { s., } \mathrm{NC}_{2}{ }_{2} \mathrm{Ph}\right)\end{array}$ & $7.30(5 \mathrm{H}, \mathrm{m} ., \mathrm{Ar}-\mathrm{H})$ \\
\hline $3 f$ & - & 2.75 & $1.15+4.25$ & $\begin{array}{l}4.8\left(2 \mathrm{H}, \mathrm{s} ., \mathrm{SCH}_{2} \mathrm{COPh}\right) \\
5.32\left(2 \mathrm{H}, \mathrm{s.}, \mathrm{NC} \underline{\mathrm{H}}_{2}(4-\mathrm{FPh})\right)\end{array}$ & $\begin{array}{l}7.06(2 \mathrm{H}, \mathrm{t} ., \mathrm{H}-2+\mathrm{H}-6) \\
7.46(2 \mathrm{H}, \mathrm{q} ., \mathrm{H}-3+\mathrm{H}-5) \\
7.55\left(2 \mathrm{H}, \mathrm{t} ., \mathrm{H}-5^{\prime}+\mathrm{H}-3^{\prime}\right) \\
7.64\left(1 \mathrm{H}, \mathrm{t} . \mathrm{H}-4^{\prime}\right) \\
8.07\left(2 \mathrm{H}, \mathrm{d} ., \mathrm{H}-2^{\prime}+\mathrm{H}-6^{\prime}\right)\end{array}$ \\
\hline $3 g$ & - & 2.82 & $1.15+4.32$ & $\begin{array}{l}4.43\left(2 \mathrm{H}, \mathrm{S} ., \mathrm{SCH}_{2}(4-\mathrm{ClPh})\right) \\
5.2\left(2 \mathrm{H}, \mathrm{S} ., \mathrm{NCH}_{2}(4-\mathrm{FPh})\right)\end{array}$ & $\begin{array}{l}7.38\left(2 \mathrm{H}, \mathrm{d} ., \mathrm{H}-3^{\prime}+\mathrm{H}-5^{\prime}\right) \\
7.31(2 \mathrm{H}, \mathrm{q} ., \mathrm{H}-3+\mathrm{H}-5) \\
7.27\left(2 \mathrm{H}, \mathrm{d}, \mathrm{H}-\mathrm{2}^{\prime}+\mathrm{H}-6^{\prime}\right) \\
7.0(2 \mathrm{H}, \mathrm{t} ., \mathrm{H}-2+\mathrm{H}-6)\end{array}$ \\
\hline $3 \mathrm{~h}$ & - & 2.83 & $\begin{array}{l}1.25+4.2 \\
1.35+4.4\end{array}$ & $\begin{array}{l}3.8\left(2 \mathrm{H}, \mathrm{s} ., \mathrm{SCH}_{2} \mathrm{COOEt}\right) \\
5.3\left(2 \mathrm{H}, \mathrm{s.}, \mathrm{NC} \underline{H}_{2}(4-\mathrm{FPh})\right)\end{array}$ & $\begin{array}{l}7.46(2 \mathrm{H}, \mathrm{q} ., 3-\mathrm{H}+5-\mathrm{H}) \\
7.06(2 \mathrm{H}, \mathrm{t}, 2-\mathrm{H}+6-\mathrm{H})\end{array}$ \\
\hline $3 i$ & - & 2.8 & $1.35+4.75$ & $\begin{array}{l}4.78\left(2 \mathrm{H}, \mathrm{s} ., \mathrm{SCH}_{2} \mathrm{COPh}\right) \\
5.4\left(2 \mathrm{H}, \mathrm{s} ., \mathrm{NC}_{2}(2-\mathrm{ClPh})\right)\end{array}$ & $\begin{array}{l}\text { 6.88-7.48 (4H, m., 2-ClPh-H) } \\
\text { 7.54 (2H, t., H-5' + H-3') } \\
7.64\left(1 \mathrm{H}, \mathrm{t} . \mathrm{H}-4^{\prime}\right) \\
8.07\left(2 \mathrm{H}, \mathrm{d} ., \mathrm{H}-2^{\prime}+\mathrm{H}-6^{\prime}\right)\end{array}$ \\
\hline $3 g$ & - & 2.83 & $1.4+4.32$ & $\begin{array}{l}4.20\left(2 \mathrm{H}, \mathrm{s} ., \mathrm{SC}_{\mathrm{H}} \mathrm{CN}\right) \\
5.35\left(2 \mathrm{H}, \mathrm{s.}, \mathrm{NC} \underline{\mathrm{H}}_{2}(2-\mathrm{ClPh})\right)\end{array}$ & 6.88-7.48 (4H, m., Ar-H) \\
\hline $3 k$ & & 2.85 & $\begin{array}{l}1.25+4.2 \\
1.35+4.4\end{array}$ & $\begin{array}{l}3.92\left(2 \mathrm{H}, \mathrm{s} ., \mathrm{SCH}_{2} \mathrm{COOEt}\right) \\
5.37\left(2 \mathrm{H}, \mathrm{s.}, \mathrm{NCH}_{2}(2-\mathrm{ClPh})\right)\end{array}$ & 7.9-7.5 (4H, m., Ar-H) \\
\hline 31 & $8.6(\mathrm{NH})$ & 2.85 & $1.42+4.35$ & $\begin{array}{l}5.3\left(2 \mathrm{H}, \mathrm{s} ., \mathrm{NCH}_{2}(2-\mathrm{ClPh})\right) \\
4.1\left(2 \mathrm{H}, \mathrm{s} ., \mathrm{SCH}_{2} \mathrm{CO}\right) \\
4.32\left(2 \mathrm{H}, \mathrm{s} ., \mathrm{NHCH}_{2} \mathrm{Ph}\right) \\
\end{array}$ & $6.9-7.5(9 \mathrm{H}, \mathrm{m} ., \mathrm{Ar}-\mathrm{H})$ \\
\hline $3 m$ & - & 2.83 & $1.34+4.31$ & $\begin{array}{l}4.35\left(2 \mathrm{H}, \mathrm{S} ., \mathrm{SCH}_{2} \mathrm{CN}\right) \\
5.27\left(2 \mathrm{H}, \mathrm{s.}, \mathrm{NCH}_{2}(3-\mathrm{ClPh})\right)\end{array}$ & 7.2-7.4 (4H, m., Ar-H) \\
\hline $3 n$ & - & 2.81 & $1.38+4.6$ & $\begin{array}{l}4.27\left(2 \mathrm{H}, \mathrm{s} ., \mathrm{SCH}_{2} \mathrm{COPh}\right) \\
5.42\left(2 \mathrm{H}, \mathrm{s} ., \mathrm{NCH}_{2}(3-\mathrm{ClPh})\right)\end{array}$ & 6.85-8.1 (9H, m., Ar-H) \\
\hline 30 & - & 2.84 & $1.38+4.22$ & $\begin{array}{l}4.45\left(2 \mathrm{H}, \text { s., } \mathrm{NHCH}_{2}(4-\mathrm{ClPh})\right) \\
5.25\left(2 \mathrm{H}, \mathrm{s} ., \mathrm{NC}_{2}(3-\mathrm{ClPh})\right)\end{array}$ & 7.10-7.45 (8H, m., Ar-H) \\
\hline $3 p$ & - & 2.79 & $\begin{array}{l}1.25+4.2 \\
1.37+4.3\end{array}$ & $\begin{array}{l}\left.\text { 3.98(2H, s., } \mathrm{SCH}_{2} \mathrm{COOEt}\right) \\
5.3\left(2 \mathrm{H}, \mathrm{s.}, \mathrm{NC}_{2}(3-\mathrm{ClPh})\right)\end{array}$ & 7.2-7.4 (4H, m., Ar-H ) \\
\hline $3 q$ & - & 2.65 & $\begin{array}{l}1.25+4.3 \\
1.35+4.4 \\
\end{array}$ & $\begin{array}{l}\left.\text { 4.05(2H, s., } \mathrm{SCH}_{2} \mathrm{COOEt}\right) \\
5.32\left(2 \mathrm{H}, \mathrm{s} ., \mathrm{NC}_{2}(3,4-\mathrm{diCl}-\mathrm{Ph})\right)\end{array}$ & 7.3-7.6 (3H, m., Ar-H) \\
\hline $3 r$ & $8.7(\mathrm{NH})$ & 2.82 & $1.42+4.3$ & $\begin{array}{l}4.05\left(2 \mathrm{H}, \mathrm{s} ., \mathrm{SCH}_{2} \mathrm{CO}\right) \\
5.32\left(2 \mathrm{H}, \text { s., } \mathrm{NHCH}_{2} \mathrm{Ph}\right) \\
4.3\left(2 \mathrm{H}, \text { s., } \mathrm{NCH}_{2}(3,4-\text { diCl-Ph })\right)\end{array}$ & 7.2-7.65 (5H, m., Ar-H) \\
\hline
\end{tabular}


Table 2 continued

\begin{tabular}{|c|c|c|c|c|c|}
\hline 1 & 2 & 3 & 4 & 5 & 6 \\
\hline $3 s$ & 7.9(NH) & 2.8 & $1.32+4.3$ & $\begin{array}{l}\text { 1.1-1.8 (10 H, m., cyclohexyl) } \\
3.85\left(2 \mathrm{H}, \mathrm{s} \text {., } \mathrm{SC} \underline{\mathrm{H}}_{2} \mathrm{CO}\right) \\
5.3\left(2 \mathrm{H}, \mathrm{S} ., \mathrm{NC} \underline{\mathrm{H}}_{2} \mathrm{Ph}\right) \\
3.55(1 \mathrm{H}, \mathrm{NH}-\mathrm{C} \underline{\mathrm{H}} \text { cyclohexyl) }\end{array}$ & 7.37-7.65 (3H, m., Ar-H) \\
\hline $3 t$ & $8.62(\mathrm{NH})$ & 2.81 & $1.38+4.3$ & $\begin{array}{l}4.07\left(2 \mathrm{H}, \mathrm{s} ., \mathrm{SCH}_{2} \mathrm{CO}\right) \\
4.22\left(2 \mathrm{H}, \mathrm{t} ., \mathrm{NC}_{2} \mathrm{CH}_{2} \mathrm{Ph}\right) \\
4.34\left(2 \mathrm{H}, \mathrm{s.}, \mathrm{NHC} \underline{\mathrm{H}}_{2} \mathrm{Ph}\right) \\
3.03\left(2 \mathrm{H} \text { t., } \mathrm{NCH}_{2} \mathrm{C}_{2} \underline{\mathrm{H}}_{2} \mathrm{Ph}\right)\end{array}$ & $7.30(10 \mathrm{H}, \mathrm{m} ., \mathrm{Ar}-\mathrm{H})$ \\
\hline $3 u$ & 7.95(NH) & 2.83 & $1.3+4.2$ & $\begin{array}{l}\text { 1.1-1.9 (10 H, m., cyclohexyl) } \\
\text { 3.97 }\left(2 \mathrm{H}, \mathrm{s} \text {., } \mathrm{SC}_{2} \mathrm{CO}\right) \\
4.22\left(2 \mathrm{H}, \mathrm{t} ., \mathrm{NC}_{2} \mathrm{CH}_{2} \mathrm{Ph}\right) \\
3.03\left(2 \mathrm{H}, \mathrm{t} ., \mathrm{NCH}_{2} \mathrm{CH}_{2} \mathrm{Ph}\right) \\
3.55(1 \mathrm{H}, \mathrm{m} ., \mathrm{NHCH} \text { cyclohexyl })\end{array}$ & 7.3-7.4 (5H, m., Ar-H) \\
\hline
\end{tabular}

Table 3

Data of IR-spectra of ethyl

5-methyl-2-(alkylthio)-4-oxo-3,4-dihydrothieno[2,3-d]pyrimidine-6-carboxylate derivartives 3

\begin{tabular}{|c|c|c|c|c|c|}
\hline \multirow[b]{2}{*}{ Compound } & \multicolumn{5}{|c|}{ Wavenumber, $\mathrm{v}, \mathrm{cm}^{-1}$} \\
\hline & $v \mathrm{~N}-\mathrm{H}$ & $\vee C-H$ & $\vee C \equiv N$ & $\vee C=O$ & $\begin{array}{l}\vee C=N \\
\vee C=C\end{array}$ \\
\hline 1 & 2 & 3 & 4 & 5 & 6 \\
\hline $3 a$ & - & $\begin{array}{lll}3085 & 3066 & 3032 \\
2976 & 2973 & 2886\end{array}$ & - & 17111683 & $\begin{array}{c}1537 \quad 1540 \quad 1470 \\
1390 \quad 1377 \quad 1363 \\
1336 \quad 1324\end{array}$ \\
\hline $3 b$ & - & $\begin{array}{c}306330343007 \\
2983 \quad 2914\end{array}$ & - & 1686 & 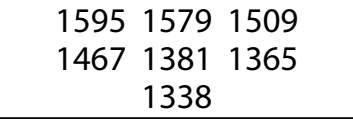 \\
\hline $3 c$ & - & $\begin{array}{c}306330332992 \\
296929302919 \\
28682709\end{array}$ & - & 17181686 & $\begin{array}{lll}1594 & 1540 & 1509 \\
1471 & 1406 & 1388 \\
1372 & 1358 & 1335\end{array}$ \\
\hline $3 d$ & 3277 & $\begin{array}{c}309130332989 \\
29312853\end{array}$ & - & $\begin{array}{c}17151681 \\
1637\end{array}$ & $\begin{array}{c}156115161468 \\
1393137613631339 \\
1309\end{array}$ \\
\hline $3 e$ & & $\begin{array}{ll}2976 & 2938 \\
2738 & 2677\end{array}$ & - & 17441697 & $\begin{array}{cc}158615381508 \\
1469 & 13741335 \\
1302 & \end{array}$ \\
\hline $3 f$ & - & 29812909 & - & 17191688 & $\begin{array}{lll}1510 & 1469 & 1449 \\
1364 & 1338 & 1311 \\
\end{array}$ \\
\hline $3 g$ & - & 299129722933 & - & 17151686 & $\begin{array}{llll}1603 & 1540 & 1509 \\
1490 & 1471 & 1444 \\
1433 & 1406 & 1387\end{array}$ \\
\hline $3 \mathrm{~h}$ & - & 306729782935 & - & 17221692 & $\begin{array}{lll}1604 & 1540 & 1510 \\
1470 & 1434 & 1388 \\
1377 & 1365 & 1338 \\
& 1319 & \\
\end{array}$ \\
\hline $3 i$ & - & 305929732924 & & 17151686 & $\begin{array}{lll}1579 & 1537 & 1509 \\
1467 & 1448 & 1365 \\
1326 & 1293 & 1251\end{array}$ \\
\hline $3 g$ & - & $\begin{array}{c}306229772941 \\
2902\end{array}$ & 2250 & 1718 & $\begin{array}{lll}1594 & 1591 & 1469 \\
1443 & 1376 & 1334 \\
\end{array}$ \\
\hline $3 \mathrm{k}$ & - & 306429802936 & - & 17191691 & $\begin{array}{lll}1537 & 1511 & 1468 \\
1443 & 1402 & 1385 \\
1368 & 1335 & 1315\end{array}$ \\
\hline 31 & 3288 & $\begin{array}{c}306430292989 \\
29292872\end{array}$ & - & 17171678 & $\begin{array}{lll}1517 & 1468 & 1443 \\
1391 & 1376 & 1332\end{array}$ \\
\hline $3 m$ & - & 29912942 & 2250 & 17101687 & $\begin{array}{r}151714731434 \\
1375\end{array}$ \\
\hline
\end{tabular}


Table 3 continued

\begin{tabular}{|c|c|c|c|c|c|}
\hline 1 & 2 & 3 & 4 & 5 & 6 \\
\hline $3 m$ & - & 29912942 & 2250 & 17101687 & 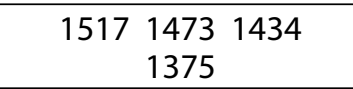 \\
\hline $3 n$ & - & $\begin{array}{c}308330642980 \\
29202871\end{array}$ & - & 1683 & $\begin{array}{lll}1597 & 1579 & 1509 \\
1464 & 1435 & 1396 \\
1378 & 1370 & 1355 \\
\end{array}$ \\
\hline 30 & - & 29792937 & - & 17191692 & $\begin{array}{llll}1595 & 1573 & 1537 \\
1510 & 1493 & 1468 \\
1436 & 1412 & 1376 \\
1358 & 1334 & 1314 \\
& 1298 \\
\end{array}$ \\
\hline $3 p$ & - & $\begin{array}{l}29882933 \\
29062872\end{array}$ & - & 17211693 & $\begin{array}{cccc}1542 \quad 1510 & 1473 \\
1437 \quad 1388 & 1377 \quad 1366 \\
1336 & 1309 & \\
\end{array}$ \\
\hline $3 q$ & - & 298129312873 & - & 17191689 & $\begin{array}{lll}1560 & 1537 & 1509 \\
1469 & 1432 & 1400 \\
\end{array}$ \\
\hline $3 r$ & 3263 & $\begin{array}{c}308930312981 \\
29352871\end{array}$ & - & 17171681 & $\begin{array}{llll}1562 & 1538 & 1511 \\
1469 & 1431 & 1399 \\
1366 & 1350 & 1331 \\
\end{array}$ \\
\hline $3 s$ & 3273 & $\begin{array}{l}30932986 \\
29332854 \\
\end{array}$ & - & 17171680 & $\begin{array}{llll}1562 & 1517 & 1470 \\
1394 & 1375 & 1352 \\
\end{array}$ \\
\hline $3 t$ & 3271 & $\begin{array}{lll}3066 & 3027 & 2990 \\
2932 & 2869 & 2285 \\
\end{array}$ & - & 17151681 & $\begin{array}{lll}1604 & 1586 & 1552 \\
1533 & 1511 & 1468 \\
\end{array}$ \\
\hline $3 u$ & 3980 & $\begin{array}{c}309030242986 \\
29322854\end{array}$ & - & 17181687 & $\begin{array}{llll}1561 & 1516 & 1468 \\
1453 & 1396 & 1377 \\
1366 & 1337 & 1303 \\
\end{array}$ \\
\hline
\end{tabular}

Table 4

Antimicrobial properties of ethyl

5-methyl-2-(alkylthio)-4-oxo-3,4-dihydrothieno[2,3-d]pyrimidine-6-carboxylate derivartives 3

\begin{tabular}{|c|c|c|c|c|c|c|}
\hline \multirow[b]{2}{*}{ Compound } & \multicolumn{6}{|c|}{$\begin{array}{l}\text { The growth inhibition zone diameter, } \mathrm{mm} \\
\text { experiments were performed in triplicate, } \mathrm{n}=3\end{array}$} \\
\hline & $\begin{array}{c}\text { Staphylococcus } \\
\text { aureus } \\
\text { ATCC } 25923\end{array}$ & $\begin{array}{l}\text { Escherichia coli } \\
\text { ATCC } 25922\end{array}$ & $\begin{array}{c}\text { Pseudomonas } \\
\text { aeruginosa } \\
\text { ATCC } 27853\end{array}$ & $\begin{array}{l}\text { Proteus vulgaris } \\
\text { ATCC } 4636\end{array}$ & $\begin{array}{l}\text { Basillus subtilis } \\
\text { ATCC } 6633\end{array}$ & $\begin{array}{c}\text { Candida } \\
\text { albicans } \\
\text { ATCC } 653 / 885\end{array}$ \\
\hline $3 a$ & $15,16,15$ & $15,14,15$ & $17,17,16$ & $16,15,14$ & $17,16,16$ & $20,19,18$ \\
\hline $3 b$ & $14,15,14$ & $16,17,16$ & $17,17,16$ & $15,16,16$ & $18,19,18$ & $17,16,17$ \\
\hline $3 c$ & $14,15,14$ & $14,15,14$ & $13,12,13$ & $12,12,13$ & $17,17,17$ & $20,21,22$ \\
\hline $3 d$ & $14,15,14$ & $14,15,14$ & $13,12,13$ & $12,12,13$ & $17,17,17$ & $20,21,22$ \\
\hline $3 e$ & $25,22,23$ & $15,15,16$ & $17,18,18$ & $16,15,16$ & $18,19,20$ & $24,22,22$ \\
\hline $3 f$ & $15,15,14$ & $14,13,14$ & $16,16,17$ & $15,14,14$ & $16,16,16$ & $14,14,14$ \\
\hline $3 g$ & $15,14,15$ & $18,17,17$ & $16,17,16$ & $15,15,15$ & $18,19,19$ & $20,21,20$ \\
\hline $3 \mathrm{~h}$ & $17,18,18$ & $13,13,14$ & $15,15,15$ & $13,14,13$ & $15,16,16$ & $17,18,17$ \\
\hline $3 i$ & $14,15,15$ & $12,13,13$ & $13,12,13$ & $12,12,13$ & $15,15,15$ & $19,18,18$ \\
\hline $3 g$ & $18,18,19$ & $16,15,15$ & $16,18,17$ & $15,15,16$ & $17,18,18$ & $16,16,17$ \\
\hline $3 \mathrm{k}$ & $14,15,15$ & $17,18,17$ & $15,17,17$ & $13,14,14$ & $16,15,16$ & $16,17,17$ \\
\hline 31 & $15,15,15$ & $16,15,16$ & $14,13,14$ & $12,13,13$ & $14,15,14$ & $20,19,19$ \\
\hline $3 m$ & $18,17,18$ & $16,16,17$ & $16,16,16$ & $15,16,14$ & $19,18,18$ & $17,16,16$ \\
\hline $3 n$ & $14,15,15$ & $13,13,13$ & $14,13,14$ & $14,13,13$ & $16,15,16$ & $19,20,19$ \\
\hline 30 & $14,14,15$ & $16,17,17$ & $22,21,20$ & $17,17,18$ & $18,19,19$ & $23,24,23$ \\
\hline $3 p$ & $15,15,15$ & $13,14,14$ & $15,16,16$ & $14,13,14$ & $15,16,17$ & $18,19,19$ \\
\hline $3 q$ & $14,15,15$ & $14,13,14$ & $14,14,14$ & $12,13,12$ & $15,17,16$ & $14,15,14$ \\
\hline $3 r$ & $15,16,15$ & $14,13,13$ & $20,18,19$ & $16,17,16$ & $16,15,16$ & $18,19,19$ \\
\hline $3 \mathrm{~s}$ & $15,16,15$ & $14,14,13$ & $20,20,21$ & $15,16,16$ & $15,16,17$ & $22,21,22$ \\
\hline $3 t$ & $15,14,14$ & $14,13,14$ & $16,17,16$ & $15,14,14$ & $15,16,16$ & $20,19,19$ \\
\hline $3 u$ & $15,14,15$ & $14,14,14$ & $15,14,14$ & $13,13,13$ & $16,17,16$ & $17,18,17$ \\
\hline
\end{tabular}


Thus, it is obvious that introduction in position 3 of chlorinated benzyl substituents or the derivative of thioacetic acid in position 2 of ethyl 5-methyl-4oxo-3,4-dihydrothieno[2,3-d]pyrimidine-6-carboxylate increases the antimicrobial properties of the compound. It is noteworthy that ethers of thioacetic acid (namely compounds $\mathbf{3 h}, \mathbf{3 k}, \mathbf{3 p}, \mathbf{3 q}$ ) show a low activity, which indicates the importance of the $\mathrm{OH}$ or $\mathrm{NH}$-acid fragment for compound $\mathbf{3}$ to be active as an antimicrobial agent.

\section{Experimental Part}

\section{Chemical research}

The melting points $\left({ }^{\circ} \mathrm{C}\right)$ were measured with a Koeffler melting point apparatus and were not corrected. IR spectra were recorded on Bruker Tensor 27 spectrometer in $\mathrm{KBr} .{ }^{1} \mathrm{H}$ NMR spectra were recorded on Bruker DRX-500 (500 MHz) spectrometers in DMSO- $d_{6}$ using TMS as an internal standard (chemical shifts are in ppm).

Diethyl methyl-5-\{[(methylthio)carbonothioyl] amino\}-2,4-thiophenedicarboxylate 1 has been obtained by the known method $[4,5]$.

General method for synthesis of compounds 3

To $2 \mathrm{~g}(0.0028 \mathrm{~mol})$ of diethyl methyl-5-\{[(methylthio)carbonothioyl] amino\}-2,4-thiophenedicarboxylate $10.004 \mathrm{~mol}$ of the corresponding primary amine was added. The mixture was diluted with DMF and stirred at reflux until the release of methylthiol occurred (about 2-5 hours). Then the reaction mixture was cooled and diluted with water. The mixture obtained was acidified with $1 \mathrm{~mL}$ of the concentrated hydrochloric acid and the precipitate formed was filtered and thoroughly washed with a lot of water. Product 2 was additionally purified by reflux in 2-propanol.
To compound $20.9 \mathrm{mmol}$ of DMF, $1 \mathrm{mmol}$ of triethylamine were added together with $1 \mathrm{mmol}$ of an alkylating agent and the mixture was stirred at $70^{\circ} \mathrm{C}$ for 8-12 hours. After that the reaction mixture was cooled and diluted with water. The precipitate of product $\mathbf{3}$ formed was filtered and crystallized forming 2-propanol.

\section{Research of the antimicrobial activity}

According to the WHO recommendations $[6,7]$ the following test-strains were used: Staphylococcus aureus ATCC 25923, Esherichia coli ATCC 25922, Pseudomonas aeruginosa ATCC 27853, Proteus vulgaris ATCC 4636, Bacillis subtilis ATCC 6633, Candida albicans ATCC653/885. The bacterial concentration was $10^{7} \mathrm{CFU} / \mathrm{mL}$ (determined by McFarland standard). Overnight cultures kept for $18-24 \mathrm{~h}$ at $36^{\circ} \mathrm{C} \pm 1^{\circ} \mathrm{C}$ were used. The bacterial suspension was inoculated onto the entire surface of a Mueller-Hinton agar (Dagestan Scientific Research Institute of Nutrient Media). The compounds were introduced to the wells in the form of DMSO solution in concentrations of $100 \mu \mathrm{g} / \mathrm{mL}$; the open wells were filled with $0.3 \mathrm{~mL}$ of the solution.

\section{Conclusions}

The synthesis of novel derivatives of ethyl 5-methyl-2-(alkylthio)-4-oxo-3,4-dihydrothieno[2,3-d]pyrimidine-6-carboxylates has been carried out and the compounds obtained have been screened for the antimicrobial activity. As the result, it has been found that introduction of chlorinated benzyl substituents in position 3 of thieno[2,3-d]pyrimidine system or the moiety of thioacetic acid in position 2 promotes the antimicrobial activity, while $\mathrm{OH}$ - or $\mathrm{NH}$-acidic fragment of thioacetic acid derivative increases the antimicrobial activity of a molecule.

\section{References}

1. Пат. US 2007197551 (2007) // Заявл.: 25.02.2005; опубл.: 23.08.2007. http://worldwide.espacenet.com/ publicationDetails $/$ biblio $? F T=D \&$ date $=20070823 \& D B=E P O D O C \& l o c a l e=e n_{-} E P \& C C=U S \& N R=2007197551$ $A 1 \& K C=A 1 \& N D=4$.

2. Hafez H.N., El-Gazzar A.-R.B.A., Nawwar G.A.M. // Eur. J. Med. Chem. - 2010. - Vol. 45, №4. - P. 1485-1493.

3. Hussein H.A.R. // Phosphorus, Sulfur, Silicon and Relat. Elem. - 2007. - Vol. 182, №9. - P. 2069-2085.

4. Ivashchenko A.V., Kovalenko S.M., Tkachenko O.V., Parkhomenko O.O. // J. Comb. Chem. - 2004. - Vol. 6, №4. - P. 573-583.

5. Kovalenko S.N., Vlasov S.V., Fedosov A.I., Chernykh V.P. // J. of Org. and Pharmac. Chem. - 2007. - Vol. 5, №3. - P. 34-40.

6. Бактеріологічний контроль поживних середовищ. Інформаційний лист МОЗ України №05.4.1/1670. К., 2001.

7. Волянський Ю.Л., Грищенко І.С., Широбоков В.П. та ін. Вивчення специбічної активності антимікробних лікарських засобів: Метод. рекоменд. - К., 2004. - 38 с.

8. Некрасова Л.С., Свита В.М., Глушкевич Т.Г. та ін. Визначення чутливості мікроорганізмів до антибактеріальних препаратів: Метод. вказівки. - К., 2007. - 79 с.

Надійшла до редакції 08.01.2013 p. 